form should be performed routinely in daily rheumatological practise including radiological assessment as well as standard evaluation of peripheral joints. Our data suggest that there is a necessity to create complex index which includes assessment of tendons/ entheses/peripheral joints for patients with JIA.

\section{A10.9 EVIDENCE FOR PROGRESSIVE REDUCTION AND LOSS OF TELOCYTES IN THE DERMAL CELLULAR NETWORK OF SYSTEMIC SCLEROSIS}

doi:10.1136/annrheumdis-2013-203224.9

1.2Mirko Manetti, ${ }^{2}$ Serena Guiducci, 'Martina Ruffo, 'Irene Rosa, 'Maria Simonetta Faussone-Pellegrini, 'Lidia Ibba-Manneschi, ${ }^{2}$ Marco Matucci-Cerinic. 'Department of Anatomy, Histology and Forensic Medicine, University of Florence, Florence, Italy; ${ }^{2}$ Department of Biomedicine, Division of Rheumatology, AOUC, and Excellence Centre for Research, Transfer and High Education DENO, The University of Florence, Florence, Italy

Background and Objectives Telocytes are a distinct population of stromal cells which have been recently identified in a wide variety of tissues and organs, including the skin. By their extremely long cytoplasmic processes telocytes may act as supporting cells and form a scaffold to define the correct three-dimensional organisation of tissues/organs during pre-natal life, or their repair/ renewal in post-natal life. Moreover, telocytes may influence the transcriptional activity of neighbouring stromal cells (fibroblasts/ myofibroblasts, mast cells), either by cell-to-cell contacts or by secreting paracrine signalling molecules, and may be implicated in tissue regeneration by cooperating with stem cell niches to form tandem cell structures. Systemic sclerosis (SSc) is a complex connective tissue disease characterised by fibrosis of the skin and internal organs. Up to now, most of the studies have focused on fibroblasts/myofibroblasts, while little is known about the possible involvement of other stromal cell types in SSc pathophysiology. In the present study, we investigated the distribution and ultrastructural features of telocytes in the skin of SSc patients compared with normal skin.

Methods Forearm skin biopsies were obtained from 24 SSc patients (13 limited cutaneous SSc (lcSSc), 11 diffuse cutaneous SSc (dcSSc)) and 10 healthy controls. Skin sections were subjected to immunoenzymatic or immunofluorescence labelling for CD34, CD31/ PECAM-1, alpha-smooth muscle actin (alpha-SMA), CD11c, CD90/ Thy-1, c-kit/CD117 and mast cell tryptase. Ultrathin sections were processed for transmission electron microscopy (TEM).

Results By an integrated immunohistochemical and TEM approach, we confirmed that telocytes were present in human dermis, where they were mainly recognisable by their typical ultrastructural features and were immunophenotypically characterised by CD34 expression. Dermal telocytes were immunophenotypically negative for CD31/PECAM-1 (endothelial cells), alpha-SMA (myofibroblasts, pericytes/vascular smooth muscle cells), CD11c (dendritic cells/macrophages), CD90/Thy-1 (fibroblasts) and c-kit/ CD117 (mast cells). In normal skin, telocytes were organised to form three-dimensional networks distributed among collagen bundles and elastic fibres, and surrounded microvessels, nerves and skin adnexa (hair follicles, sebaceous and sweat glands). Telocytes displayed severe ultrastructural damages (swollen mitochondria, cytoplasmic vacuolisation, lipofuscinic bodies) suggestive of ischaemia-induced cell degeneration and were progressively lost from the clinically affected skin of SSc patients. Telocyte damage and loss evolved differently according to $\mathrm{lcSSc} / \mathrm{dcSSc}$ subsets and early/advanced stages, being more rapid and severe in dcSSc.

Conclusions In SSc skin, the progressive loss of telocytes might i) contribute to the altered three-dimensional organisation of the extracellular matrix, ii) reduce the control of fibroblast/myofibroblast and mast cell activity, and iii) impair skin regeneration and/or repair.

\section{A10.10 EXAMINATION OF IL-6, TNF I AND II RECEPTOR DISTRIBUTION ON PERIPHERAL BLOOD MONONUCLEAR CELLS (PBMC) IN SEROPOSITIVE AND SERONEGATIVE RHEUMATOID ARTHRITIS (RA)}

doi:10.1136/annrheumdis-2013-203224.10

Martyna Skwarek, Babett Heschel, Julia Fantana, Martin Aringer. Division of Rheumatology, Department of Medicine III, University Clinical Center Carl Gustav Carus at the Technical University of Dresden, Germany

Background and Objectives Despite significant clinical overlap, genetic evidence and, in part, response to therapy distinguish seropositive $(R A+)$, i.e. rheumatoid factor $(R F)$ positive RA patients from patients with RF and anti-CCP-negative (RA-) disease. The aim of this study was to profile RA+ and RA- patients with regard to the differential expression of receptors for IL- 6 and TNF, cytokines of established importance in RA.

Materials and Methods PBMC of RA+ and RA- patients were compared to each other and to healthy individuals (HC). Most (93\%) of the RA+ patients were also positive for anti-CCP antibodies. PBMC were immediately prepared from peripheral venous blood. For determining the percentage of IL-6R $\alpha$ (CD126), gp130 (CD130), TNFR I (CD120a), and TNFR II (CD120b) positive cells, PBMC were stained with PE-labelled or control antibodies. Cells were analysed on a Becton Dickinson FACSCalibur fluorocytometer, gating for lymphocytes.

Results Disease duration (median 7 (0.04-66) versus 3.5 (0.04-6) years) and disease activity (CDAI median 15.6 (5.3-54.5) versus 13.5 (4.4-28)) were comparable between RA+ and RA- patients. Lymphocytes of RA+ and RA- patients differed in their lymphocyte expression of CD126+ and CD120b+. The percentage of CD126+ lymphocytes in RA+ was decreased in comparison with RA- (mean $\pm \mathrm{SD}, 49 \pm 14$ versus RA-58 $\pm 11, \mathrm{p}=0.05)$ and $\mathrm{HC}(59 \pm 9 \%$, $p=0.0007)$. The difference between RA- and HC was not significant. The percentage of CD130+ lymphocytes in RA+ (51 \pm 11$)$ was decreased when compared with $\mathrm{HC}(58 \pm 11 \%, \mathrm{p}=0.007)$. While the mean values for CD130 were similar between RA+ and RA- $(55 \pm 14 \%)$, the latter values were not significantly different from HC. In contrast to the IL-6 receptor, CD120b+ lymphocytes were increased in RA+ patients $(69 \pm 12 \%$ versus $R A-58 \pm 12 \%$, $p=0.04$, versus HC $57 \pm 11, p=0.0002)$. Again, the difference between RA- and HC was not significant. The percentages of CD120a + lymphocytes were low in all groups. Nevertheless, mean percentages of CD120a + lymphocytes from RA- (1.5 (0.53-2) \% versus HC (median $1(0.4-4) \% p=0.05)$ were somewhat higher than those of RA+ (median $1.1(0.3-3) \%, p=0.9)$.

Conclusions A direct comparison of (IL-6 mediated) downregulation of CD126 and (TNF mediated) upregulation of CD120b suggests that both are clearly more pronounced in RA+ than in RA-0. This was not explained by differences in disease activity.

\section{A10.11 EXPRESSION OF UNFOLDED PROTEIN RESPONSE GENES IN SYNOVIUM AND BLOOD MONONUCLEAR CELLS OF HLA-B27 POSITIVE ANKYLOSING SPONDYLITIS PATIENTS IS NOT INCREASED COMPARED TO OTHER ARTHRITIS PATIENTS AND HEALTHY CONTROLS}

doi:10.1136/annrheumdis-2013-203224.11

Barbara Neerinckx, Shea Carter, Rik J Lories. Laboratory of Tissue Homeostasis and Disease, Skeletal Biology and Engineering Research Center, Department of Development and Regeneration, KU Leuven, Belgium

Background The HLA-B27 heavy chain is prone to misfolding. Misfolded proteins give rise to endoplasmic reticulum stress and activation of the Unfolded Protein Response (UPR). The UPR is strongly activated in HLA-B27 transgenic rats, an animal model for 